\title{
Bell-type Inequalities from Separate Common Causes
}

\author{
Gerd Graßhoff $\quad$ Adrian Wüthrich* \\ History and Philosophy of Science \\ University of Bern, Switzerland
}

\begin{abstract}
In the light of recent discussions we present the main results of our project, the aim of which was to derive a Bell-type inequality from the weakest possible assumptions. A principal outcome of the project is that a Bell-type inequality can be derived from the assumption of separate common causes (Graßhoff, Portmann and Wüthrich 2005), even without the assumption of perfectly anticorrelating event types (Portmann and Wüthrich 2007). We also address the critique that in Graßhoff et al. (2005) we implicitly assume a common common cause (Hofer-Szabó forthcoming).
\end{abstract}

\section{Contents}

1 Introduction 1

2 Common causes for correlated events 2

3 A common screener-off is not a common common cause 3

4 "Genuine" separate common causes 4

4.1 Relative minimality of derivations . . . . . . . . . . . . . . . . 4

4.2 "Genuine" separate screener-offs . . . . . . . . . . . . 5

$\begin{array}{lll}5 & \text { Summary } & 6\end{array}$

\section{Introduction}

Bell-type inequalities provide predictions of observable frequencies of measurement outcomes in a particular experiment in quantum mechanics (the EPR-

\footnotetext{
* Speaker at the conference (EPSA, Madrid 2007). Title of the talk: Minimal Assumption Derivation of a Bell-Type Inequality.
} 
Bohm experiment). Although the actual experiments were conducted with polarized photons, for our purposes we can consider the Bell inequalities as having being tested in the familiar setup proposed by Bohm and Aharonov (1957), which is based on Einstein, Podolsky and Rosen's original formulation of the paradox that bears their names (EPR) (Einstein, Podolsky and Rosen 1935). We will refer to the experimental setup described by Bohm as the EPRB experiment.

In the EPRB experiment the particles of a pair in the spin singlet state are separated from each other by an arbitrary distance. We assume that one particle flies into the left wing of the experimental setting, the other particle into the right wing. Each particle's spin is measured relative to one of three directions. The individual particle measurements yield an apparently random sequence of results that are either "spin up" or "spin down" relative to the chosen measurement direction. A comparison of measurement results when parallel measurement settings are chosen shows a perfect (anti)correlation between the outcome of the measurement performed on the left particle and the outcome of the measurement performed on the right particle.

The assumption that the correlations require a local causal explanation leads to predictions (a Bell inequality) that contradict quantum mechanics as well as, by current standards, the experimental data. Thus, at least one of the assumptions needed to derive a Bell inequality must be wrong.

The argument has the form of a reductio ad absurdum: from the falsity of the conclusion, the falsity of one of the premises is inferred. The strength of the argument rests on the fact that:

- the derivation is deductive;

- all the assumptions are explicit; and

- the set of assumptions is minimal

in the sense that no subset of the assumptions implies a Bell inequality. Since derivations of Bell-type inequalities are often invoked as arguments against the causal closedness of the physical world, the derivation should above all suppose a notion of causal explanation that is "non-trivial" and "as weak as possible" (van Fraassen 1982, p. 27).

\section{Common causes for correlated events}

The event types that are observed to be correlated in a EPRB experiment are assumed, justifiably, not to stand in a direct causal relation. If we are to explain the correlations at all, we have to do so by postulating a common cause for the correlated effects. Most derivations, lacking as they do a sound theory of causal relevance, resort to Reichenbach's common-cause principle (Reichenbach 1956) providing a seemingly necessary condition for causal relevance (screening-off). The principle states that, conditional upon the instantiation of the common cause, the events in question are uncorrelated. This statistical condition is 
weaker than the demand that the common cause be sufficient for its effects, but it still captures the idea that, with the exception of the common cause, there is no reason for the event types to be correlated.

As Reichenbach explicated the screening-off condition only with reference to one pair of correlated events, it is not clear how one should apply this principle to the EPRB setup (cf. Hofer-Szabó, Rédei and Szabó 1999). Traditional derivations assume that there is a common common cause for all correlated pairs of events and, therefore, a common screener-off:

$$
p\left(L^{+} R^{-} \mid M_{i} N_{i} C\right)=p\left(L^{+} \mid M_{i} N_{i} C\right) p\left(R^{-} \mid M_{i} N_{i} C\right) \quad \forall i .
$$

This, however, is still an unjustifiably strong assumption. Nothing in Reichenbach's notion of common causes dictates that the common cause should be common to all correlated pairs. A more general application of Reichenbach's condition only demands that, for each correlated pair, there is a (possibly different) common cause. From the assumption of separate common causes, only the following screening-off condition can be justified:

$$
p\left(L^{+} R^{-} \mid M_{i} N_{i} C_{i i}\right)=p\left(L^{+} \mid M_{i} N_{i} C_{i i}\right) p\left(R^{-} \mid M_{i} N_{i} C_{i i}\right),
$$

where the common causes $C$ are indexed as being the cause of the measurement outcomes of a specific choice of measurement directions.

The $C$ indices express neither a causal nor a statistical dependence between the common causes and the measurement operations. They are simply labels to distinguish between types of events (at the source where the particles are created). The distinguished event types at the particle source are not mutually exclusive descriptions of a "total state". A common cause responsible for a correlation exhibited by a certain measurement need not occur together with the corresponding measurement. All that is required is that the screeningoff conditions hold for the common causes with respect to the measurement results. The common causes may or may not be instantiated in each run of the experiment, irrespective of which measurement event type is chosen to be instantiated. Separate common causes need not be causally relevant to the choice of measurements nor is a causal influence backwards in time from the measurement choices on the common causes required.

\section{A common screener-off is not a common com- mon cause}

Hofer-Szabó et al. (1999) claim that without the unjustified strong assumption, made by traditional derivations, of a common common cause "Bell's inequality cannot be derived" (p. 388, emph. in the original). Graßhoff et al. (2005) proved the contrary, supposing reasonable locality and other independence conditions. This derivation assumes a weaker notion of common cause explanations than traditional derivations and, therefore, provides a stronger reductio ad absurdum 
argument against the possibility of the EPRB correlations having a common cause explanation.

One could surmise, though, that the conjunction of the separate common causes just makes up a common common cause. But, in general, this is not the case, since, for instance, from

$$
p\left(A_{i} B_{i} \mid C_{i}\right)=p\left(A_{i} \mid C_{i}\right) p\left(B_{i} \mid C_{i}\right)
$$

and

$$
p\left(A_{j} B_{j} \mid C_{j}\right)=p\left(A_{j} \mid C_{j}\right) p\left(B_{j} \mid C_{j}\right)
$$

it does not follow that

$$
p\left(A_{i} B_{i} \mid C_{i} C_{j}\right)=p\left(A_{i} \mid C_{i} C_{j}\right) p\left(B_{i} \mid C_{i} C_{j}\right)
$$

Only under specific circumstances is the conjunction of separate common causes a common screener-off. For instance, in the case of a perfect correlation between $A$ and $B,(3)$ does follow from (1), as the conditional probabilities are zero or one. Thus, in the case of perfect correlations, the conjunction of the separate common causes is a common screener-off and, therefore (supposing Reichenbach's notion of common cause), could, but need not, be a common common cause (since the Reichenbach condition is, at most, a necessary condition).

Common screener-offs, however, are not common common causes, since Reichenbach took the screening-off condition to be only a necessary and not a sufficient condition for qualifying as a common cause - and rightly so. In the case of perfect correlations, the screener-offs are sufficient for the correlated events $\left(p\left(A_{i} \mid C_{i}\right)=1\right.$, for instance). The conjunction of the common causes $\left(C_{i} C_{j}\right)$ is still a sufficient condition for the effects and, therefore, a (trivial) common screener-off. But it is clear, either intuitively or given the appropriate causal theory (Graßhoff and May 2001), that $C_{j}$ (the common cause for $A_{j}$ and $B_{j}$ ), for instance, does not contribute to the correlations of $A_{i}$ and $B_{i}$ and hence is not an appropriate part of a common cause for that correlation. Sufficient conditions for events tend to include more than their causes. Only minimally sufficient conditions can qualify as causes for events (Graßhoff and May 2001).

\section{4 "Genuine" separate common causes}

\subsection{Relative minimality of derivations}

In Graßhoff et al. (2005) we do not assume a common common cause in deriving a Bell-type inequality. In this respect the derivation is weaker than traditional derivations. However, in a different respect, Clauser, Horne, Shimony and Holt's (1969) derivation, for instance, is weaker: it is not assumed, in the case of parallel measurement settings, that the outcomes are perfectly anticorrelated; on the cost that they require a common common cause. The minimality of the 
set of assumptions of Graßhoff et al. (2005) is, therefore, only relative to the group of derivations that do not assume perfect anticorrelations. A derivation of a Bell-type inequality from separate common causes without the assumption of perfect anticorrelations was published in April 2006 as an electronic preprint before being published in print (Portmann and Wüthrich 2007). A similar derivation is forthcoming (in Hofer-Szabó forthcoming).

In Portmann and Wüthrich (2007) a small deviation from perfect anticorrelation was allowed, as for instance: $p\left(L^{+} \mid R^{-} M_{i} N_{i}\right)=1-\epsilon$. Together with the assumption of separate common causes (and traditional locality and independence assumptions), an inequality was derived that is of the same form as the Clauser-Horne inequality (Clauser and Horne 1974) but less restrictive by essentially the amount $\epsilon$. Quantum mechanical predictions contradict the predictions of a common-cause model obeying the assumptions of Portmann and Wüthrich (2007) for $\epsilon \leq 2.689 \cdot 10^{-5}$. It is, however, extremely difficult to obtain such high precision in experiments confirming perfect anticorrelation, but only then is the inequality violated by the quantum mechanical predictions and experimental data. Thus, common-cause models obeying the assumptions of Portmann and Wüthrich (2007) can hardly be ruled out by empirical data.

There are, however, theoretical reasons for opposing the possibility of such a common-cause model. If the model is not to be dismissed by the violation of the inequality derived by Portmann and Wüthrich (2007), the deviation from perfect anticorrelation should be greater than $2.689 \cdot 10^{-5}$. Yet, the theoretical predictions of a deviation from perfect anticorrelation by, for instance, quantum gravity effects, are much smaller. Thus, current theories, which, contrary to standard quantum mechanics, predict a slight deviation from perfect anticorrelations, would hardly be able to explain a deviation by the required amount.

A further proviso is in order: it is an open question whether even the original (more restrictive) Clauser-Horne inequality can be derived from the assumptions of Portmann and Wüthrich (2007). In that case common-cause models obeying the assumptions of Portmann and Wüthrich (2007) would be ruled out by an empirical falsification of the Clauser-Horne inequality - as are the models considered by these authors (Clauser and Horne 1974).

\section{2 "Genuine" separate screener-offs}

Only when deviations from the perfect anticorrelations (PCORR) postulated by quantum mechanics are allowed is the conjunction of the separate screener-offs not a common screener-off. For Hofer-Szabó (forthcoming) only then are there no "implicit" common screener-offs (C-SCR), and only such derivations can provide a derivation of Bell's inequality from "genuine" separate screener-offs (SSCR). But also Graßhoff et al.'s (2005) derivation is a conclusive derivation of a Bell inequality (BELL) from separate screener-offs (and from separate common causes). The fact that, in the case of perfect correlations, the existence of a common screener-off is implied by the existence of separate screener-offs does not invalidate the statement that (together with PCORR and other assumptions $\mathrm{X})$ Bell's inequality follows from the assumption of separate screener-offs. If only 
separate common causes, the conjunction of which is not a common screener-off, counted as "genuine", then there would be no genuine separate common causes for perfect correlations.

Graßhoff et al. (2005) contains the statement that S-SCR, PCORR and X implies BELL:

$$
\text { S-SCR, PCORR, X } \rightarrow \text { BELL. }
$$

From traditional derivations it is known that

$$
\text { C-SCR, PCORR, X } \rightarrow \text { BELL. }
$$

Hofer-Szabó (forthcoming) grounds his critique on the fact that

$$
\mathrm{S}-\mathrm{SCR}, \mathrm{PCORR} \rightarrow \mathrm{C}-\mathrm{SCR} .
$$

However, the (granted) truth of this statement does not invalidate Graßhoff et al.'s (2005) claimed implication (4). Thus, in that respect (quite apart from the non-identity of common causes and screener-offs discussed above) HoferSzabó's (forthcoming) critique also does not invalidate Graßhoff et al.'s (2005) argument.

\section{Summary}

Traditional derivations of Bell-type inequalities assume, without sound justification, a common cause that is identical for all correlated pairs of events. However, a Bell-type inequality can also be derived from the weaker assumption that, for each correlated pair, there is a (possibly different) common cause. The resulting set of assumptions, one of which by reductio ad absurdum must be false, may or may not include the assumption of perfect anticorrelation with parallel measurement settings (Graßhoff et al. 2005, Portmann and Wüthrich 2007). The proof that a Bell-type inequality can be derived from separate common causes has been conjectured to be impossible (Hofer-Szabó et al. 1999, p. 388), but the argument is not invalidated by the mathematical construction of a common screener-off from the separate common causes, contrary to what, we read, is claimed in Hofer-Szabó (forthcoming).

\section{References}

Bohm, D. and Aharonov, Y.: 1957, Discussion of experimental proof for the paradox of Einstein, Rosen, and Podolsky, Phys. Rev. 108(4), 1070-1076.

Clauser, J. F. and Horne, M. A.: 1974, Experimental consequences of objective local theories, Phys. Rev. D 10(2), 526-535.

Clauser, J. F., Horne, M. A., Shimony, A. and Holt, R. A.: 1969, Proposed experiment to test local hidden-variable theories, Phys. Rev. Lett. 23(15), 880-884. 
Einstein, A., Podolsky, B. and Rosen, N.: 1935, Can quantum-mechanical description of physical reality be considered complete?, Phys. Rev. $\mathbf{4 7}(10), 777-780$.

Graßhoff, G. and May, M.: 2001, Causal Regularities, in W. Spohn, M. Ledwig and M. Esfeld (eds), Current Issues in Causation, Paderborn: Mentis, pp. $85-114$.

Graßhoff, G., Portmann, S. and Wüthrich, A.: 2005, Minimal Assumption Derivation of a Bell-type Inequality, The British Journal for the Philosophy of Science 56(4), 663-680.

Hofer-Szabó, G.: forthcoming, Separate- versus common-common-cause-type derivations of the Bell inequalities, Synthese pp. - .

Hofer-Szabó, G., Rédei, M. and Szabó, L. E.: 1999, On Reichenbach's common cause principle and Reichenbach's notion of common cause, The British Journal for the Philosophy of Science 50(3), 377-399.

Portmann, S. and Wüthrich, A.: 2007, Minimal assumption derivation of a weak Clauser-Horne inequality, Studies In History and Philosophy of Modern Physics 38(4), 844-862. Electronic preprint: quant-ph/0604216.

Reichenbach, H.: 1956, The Direction of Time, Dover Publications.

van Fraassen, B. C.: 1982, The charybdis of realism: Epistemological implications of Bell's inequality, Synthese 52(1), 25-38. 